\title{
Entwicklung eines Systems für hochpräzise Mikroverzahnungsmessungen
}

\author{
$\underline{\text { Stephan Jantzen }}^{1 \S}$, David Metz $^{2 \S}$, Martin Stein ${ }^{1}$, Karin Kniel$^{1}$, Andreas Dietzel$^{2}$ \\ 1 Physikalisch-Technische Bundesanstalt (PTB), Fachbereich Koordinatenmesstechnik, \\ Bundesallee 100, 38116 Braunschweig, Deutschland \\ 2 Technische Universität Braunschweig, Institut für Mikrotechnik (IMT), \\ Alte Salzdahlumer Str. 203, 38124 Braunschweig, Deutschland \\ $\S$ Beide Autoren haben zu gleichen Teilen zu der Arbeit beigetragen
}

\begin{abstract}
Kurzfassung
Das von uns realisierte System besteht aus drei unabhängigen Entwicklungen: dem IMT-PTB-Mikrotaster, einem Mikroinnenverzahnungsnormal und einem Mikroenvironment. Der IMT-PTB-Mikrotaster ist ein auf Silizium -Feder-Parallelogrammen basierender Taster, der in kommerzielle Koordinatenmessgeräte integriert wird, um Mikromessungen zu ermöglichen. Bei dem Mikroinnenverzahnungsnormal handelt es sich um einen von der PTB, dem nationalen Metrologieinstitut Deutschlands, kalibrierten Prüfkörper mit Moduln zwischen 0,1 mm und $1 \mathrm{~mm}$. Die dritte Systemkomponente, das Mikroenvironment, ist ein multifunktionales System, welches in Koordinatenmessgeräte integriert wird, um die Messung von Mikrostirnrädern zu verbessern und beschleunigen. Es besteht aus Aufspannung, Schutzumhausung, Reinigungs- und Sensorsystem sowie zwei Mikroskopkameras.

Der kombinierte Einsatz von Mikrotaster, -normal und -environment ermöglicht eine rückgeführte, optimierte Messung von Mikroverzahnungen auf kommerziellen Koordinatenmessgeräten. Das System ist eine kostengünstige Alternative zu Mikrokoordinatenmessgeräten.
\end{abstract}

Keywords: Fertigungsmesstechnik, Mikroverzahnung, membranbasierter isotroper Mikrotaster, Mikroenvironment, Mikroinnenverzahnungsnormal

\section{Motivation}

Die zuverlässige Messung von Mikroverzahnungen ist essentiell für deren Qualitätskontrolle in der Fertigung. Mögliche Abweichungen von der Sollgeometrie bei Verzahnungen können zu erhöhtem Verschleiß, Geräusch, Ausfall des Getriebes und verringerter Effizienz führen. Die taktile Messung von Verzahnungen ist weit verbreitet aufgrund der meist konkurrenzlosen Genauigkeit, den etablierten Technologien und der langen Entwicklungshistorie, was sich unter anderem in der umfangreichen und anerkannten Normung zeigt.

Messungen von Werkstücken mit Merkmalen im Sub-Millimeterbereich erfordern angepasste Messsysteme und -strategien. Gründe hierfür sind Schwierigkeiten bei der Handhabung, die benötigten kleineren Tastelemente und -kräfte und die hohen Anforderungen an die Kontaminationskontrolle. Aus diesem Grund haben die Physikalisch-Technische Bundesanstalt (PTB) und das Institut für Mikrotechnik der TU Braunschweig (IMT) in einem gemeinsamen Projekt ein System entwickelt, das die Herausforderungen in der Mikroverzahnungsmesstechnik überwindet.

Das entwickelte System besteht aus drei Komponenten:

- Isotroper IMT-PTB-Mikrotaster mit großen Auslenkungsbereichen

- Kalibriertes Mikroinnenverzahnungsnormal aus Hartmetall bzw. Titan

- Portables Mikroenvironment zur Prozessverbesserung und -beschleunigung

\section{IMT-PTB-Mikrotaster}

Der IMT-PTB-Mikrotaster beruht auf dem patentierten Prinzip von drei seriell angeordneten Silizium-Feder-Parallelogrammen (siehe Abbildung 1) [1]. Die Membrandicken sind in der Größenordnung von $25 \mu \mathrm{m}$. Anpassungen der einzelnen Parallelogrammgeometrien ermöglichen eine spezifische Optimierung von Isotropie und Steifigkeit in allen Raumrichtungen.

Der Parallelogrammaufbau ist die mikrotechnische Weiterentwicklung der in der Koordinatenmesstechnik etablierten Tastkopfaufbauten für Koordinatenmessgeräte (KMG) [2]. Die mikro- 


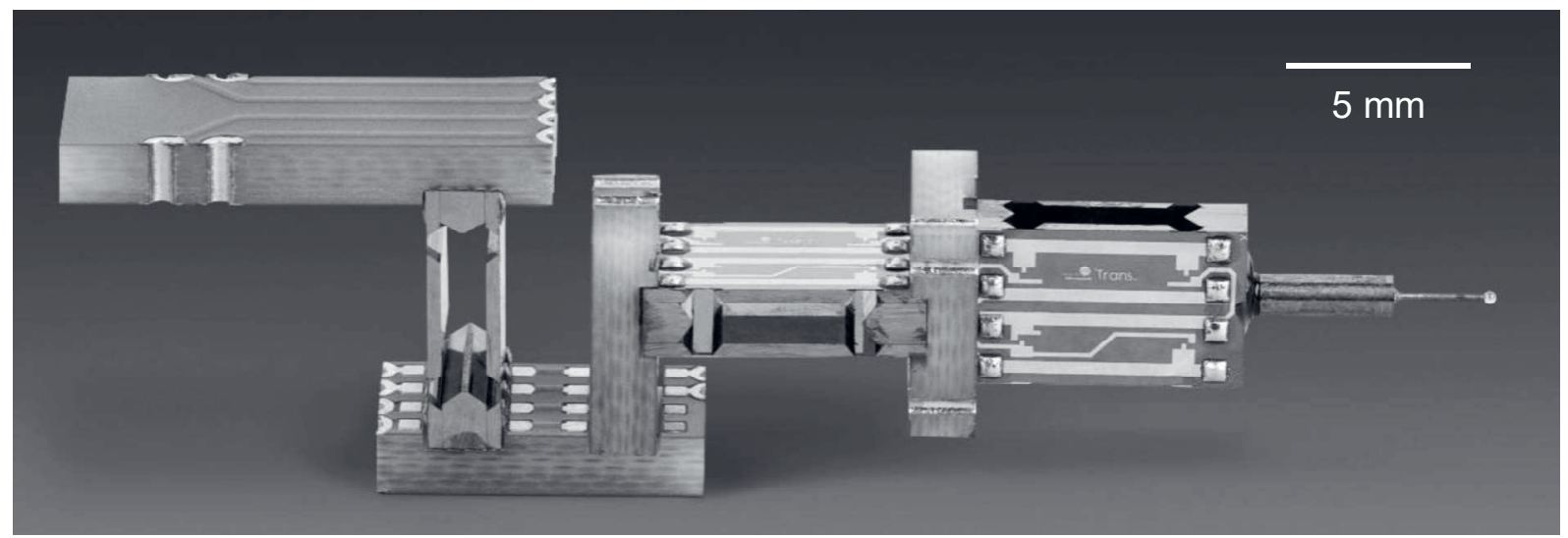

Abbildung 1: Aufbau des IMT-PTB-Mikrotasters (ohne Gehäuse). Die Steifigkeit des Tasters beträgt circa $0,3 \mathrm{mN} \cdot \mu \mathrm{m}^{-1}$. Die Verbindungsstücke zwischen den Parallelogrammen führen die elektrischen Signale weiter bis zur Kontaktierung am Gehäuse.

technische Adaption nutzt die Vorteile der Siliziumtechnik und integriert die Sensoren in die Festkörpergelenke (siehe Abbildung 2) [3]. Damit wird eine Miniaturisierung des Funktionsprinzips erreicht.

In die Siliziumfestkörpergelenke eindiffundierte Piezowiderstände messen die Auslenkung der Parallelogramme mithilfe von Wheatstone-Brücken. Mit dem neuartigen Prinzip sind Auslenkungen bis zu $\pm 400 \mu \mathrm{m}$ möglich, was eine deutliche Verbesserung zu vergleichbaren membranbasierten Mikrotasterdesigns ist [4]. Der Taster kann mit Tastkugeldurchmessern zwischen $50 \mu \mathrm{m}$ und $1 \mathrm{~mm}$ verwendet werden. Die exzellente Isotropie des Tasters aufgrund seines seriellen Parallelogrammaufbaus bietet messtechnische Vorteile, zum Beispiel wenn schräge Flächen angetastet werden.

Der IMT-PTB-Mikrotaster wurde in ein kommerzielles Verzahnungsmessgerät, die P40 der Firma Klingelnberg, erfolgreich integriert und experimentell verifiziert [5, 6, 7]. Die Integration läuft über einen in dem Messgerät vorhandenen A/D-Wandler. Die Messgerätsoftware ist angepasst, um die Auslenkungssignale des Mikrotasters anstelle der des Standardtastkopfs auszuwerten. Messungen laufen daher mit der Standardsoftware und den gleichen Messroutinen wie in der Standardkonfiguration, was eine sehr gute Bedienbarkeit ermöglicht.

Der IMT-PTB-Mikrotaster wurde anhand von Vergleichsmessungen an kalibrierten Prüfkörpern (Kugel, Würfel und dem Mikroinnenverzahnungsnormal (siehe folgenden Abschnitt)) verifiziert [8]. Die Messergebnisse zeigen Differenzen zu den Kalibrierwerten im Sub-Mikrometerbereich, was den Erfolg von Design und Realisierung bestätigt.

\section{Mikroinnenverzahnungsnormal}

Das in der PTB entwickelte Mikroinnenverzahnungsnormal [9] ermöglicht eine Erweiterung der Rückführung von Verzahnungsmessungen (zum Beispiel mit dem Substitutionsverfahren, siehe auch DIN EN ISO 15530-3).

Vier verschiedene Moduln sind an dem Prüfkörper realisiert: $1 \mathrm{~mm}, 0,5 \mathrm{~mm}, 0,2 \mathrm{~mm}$ und $0,1 \mathrm{~mm}$ (siehe Abbildung 3). Die Kalibrierung wurde auf dem Mikro-Koordinatenmessgerät Zeiss F25 mit einem Rubintastkugeldurchmesser von $120 \mu \mathrm{m}$ durchgeführt. Die erweiterten Messunsicherheiten $(k=2)$ der taktilen Kalibrierung liegen zwischen $0,3 \mu \mathrm{m}$ und $0,6 \mu \mathrm{m}$ für die Winkel-, Form- und Gesamtabweichungen an den gemessenen Profilen und Flankenlinien.

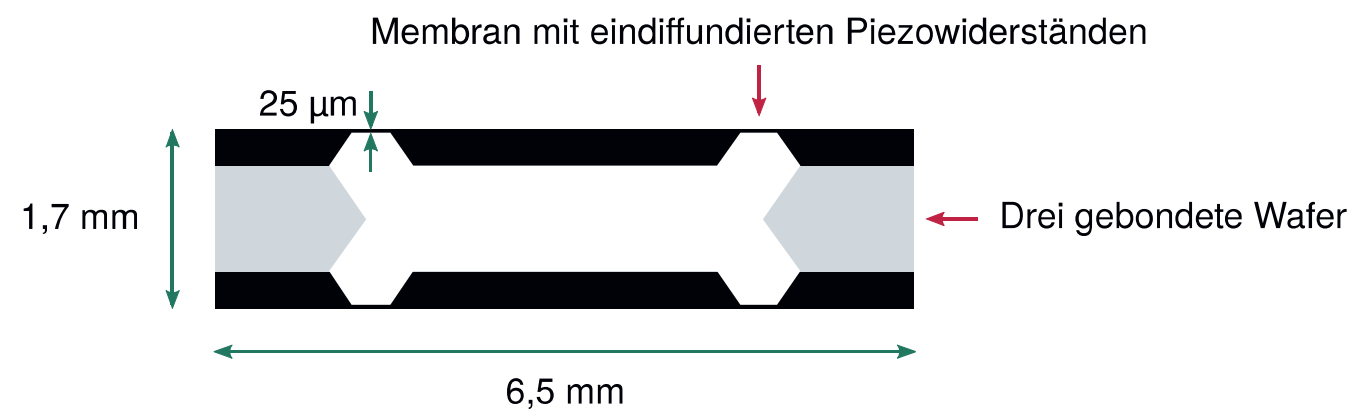

Abbildung 2: Querschnitt eines einzelnen Parallelogramms. Durch die Geometrie besitzen die Parallelogramme eine geringe Steifigkeit in einer Raumrichtung (Das Verhältnis der Steifigkeiten zwischen Vorzugsrichtung und den beiden anderen Achsen beträgt bis zu 1:300). 


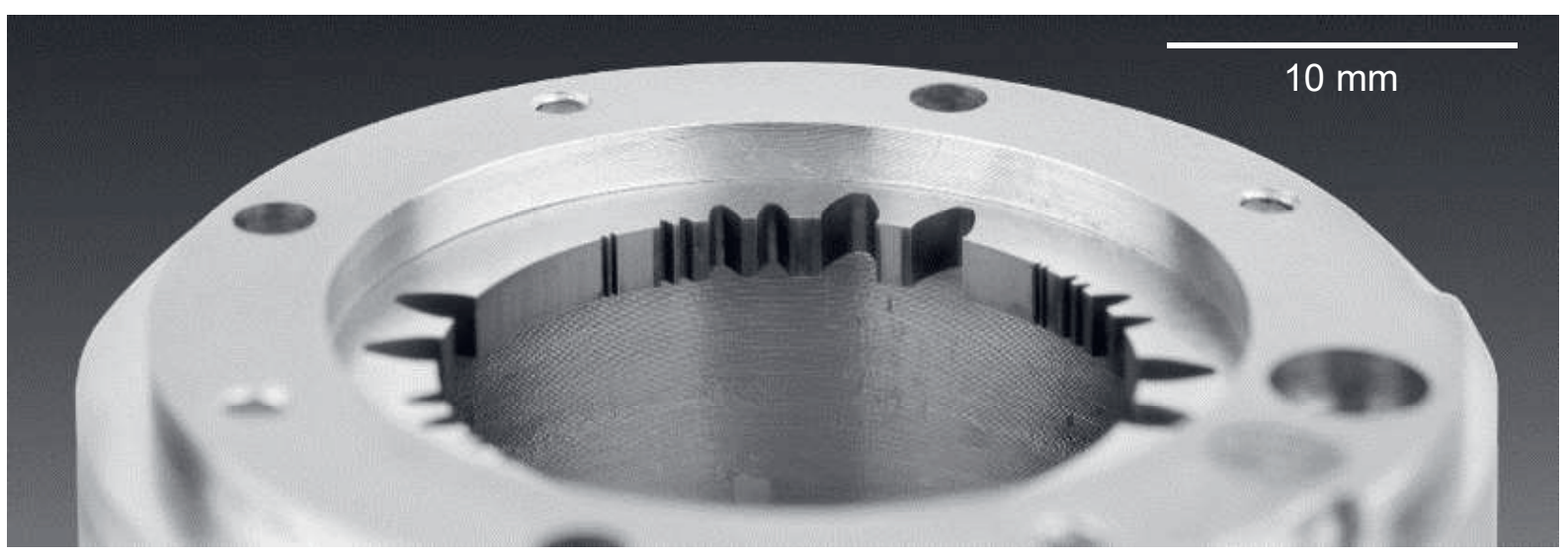

Abbildung 3: Detailaufnahme des Mikroinnenverzahnungsnormals.

Die Messunsicherheiten wurden GUM-konform ermittelt [10]. Die Eingangsparameter wurden mithilfe von Experimenten, Monte-Carlo-Simulationen und Expertenwissen bestimmt.

Der Prüfkörper besitzt einen Fußkreisdurchmesser von $20 \mathrm{~mm}$ und eine Zahnbreite von $2 \mathrm{~mm}$. Die Zahnbreite orientiert sich an der Schaftlänge des Tasters. Ein möglicher Ansatz für beliebig große Zahnbreiten bei limitierten Mikrotaster-Schaftlängen ist das Stapeln und Verbinden von mehreren einzeln kalibrierten Prüfkörpern.

Zwei Versionen des Normals wurden in der PTB mit Drahterosion gefertigt. Eine Version besteht aus Hartmetall und ist aufgrund seiner Materialeigenschaften für taktile Messungen gedacht. Allerdings ist die Oberflächenhärte, die wir durch das Fertigungsverfahren zurzeit erreichen noch verbesserungswürdig. Die zweite Version besteht aus Titan und ist damit auch geeignet für Messungen mit Computertomografie. Beide Versionen wurden kalibriert.

Nach der taktilen Kalibrierung haben wir Vergleichsmessungen mit den Prüfkörpern mit unterschiedlichen Messprinzipien und -systemen durchgeführt. Hierbei ermöglichen die Computertomografie sowie die optischen Verfahren mittels Durchlicht und Fokusvariation auch die Messung des 0,1 mm Moduls.

Die Messung des Normals mit Computertomografie lieferte eine Voxelgröße von $(22,7 \mu \mathrm{m})^{3}$ bedingt durch das große Verhältnis von Außendurchmesser ( $\varnothing 40 \mathrm{~mm}$ ) zu Zahngrößen (Moduln von $1 \mathrm{~mm}$ bis $0,1 \mathrm{~mm}$ ). Eine Messung bestehend aus 2500 Projektionen dauerte $167 \mathrm{Mi}-$ nuten. Die Abweichungen zu den Kalibrierwerten lagen im Bereich von $4 \mu \mathrm{m}$.

Bei der Messung mit Fokusvariation konnte nicht der ganze Prüfkörper erfasst werden (wie es bei Computertomografie möglich ist), sondern lediglich die Innenkontur und Teile der oberen Planfläche. Daher musste die Definition des Werkstückkoordinatensystems im Vergleich zu der taktilen Kalibrierung angepasst werden. An einzelnen Stellen konnte keine Oberflächeninformation erfasst werden, was zu Fehlstellen führte. Eine Messung dauerte 106 Minuten. Die Abweichungen zu den Kalibrierwerten lagen im Bereich von $4 \mu \mathrm{m}$ und waren damit vergleichbar mit den Computertomografie-Ergebnissen.

Die taktilen Vergleichsmessungen wurden auf der Klingelnberg P40 durchgeführt. Hierbei verwendeten wir das Standardtastsystem als auch den IMT-PTB-Mikrotaster. Die Messstrategie wurde - soweit möglich - von der taktilen Kalibrierung übernommen. Die Messzeiten betrugen beispielsweise circa 20 Minuten sechs Lücken des $0,5 \mathrm{~mm}$ Moduls inklusive Prüfbunde. Die Abweichungen zu den Kalibrierwerten lagen im Sub-Mikrometer-Bereich.

Insgesamt bewerten wir die Vergleichsmessungen positiv. Die Ergebnisse und Erfahrungen verdeutlichen die Anforderungen, Vor- und Nachteile der jeweiligen Messverfahren [11].

\section{Mikroenvironment}

Das portable Mikroenvironment wurde entwickelt, um das Messsystem - bestehend aus IMT-PTB-Mikrotaster und Klingelnberg P40 hinsichtlich der Praktikabilität für die Mikro-Koordinatenmesstechnik zu verbessern. Das Mikroenvironment besteht aus Lösungen für Aufspannung, Reinigung, Kontaminationskontrolle und Prozessüberwachung.

Die Aufspannung für Mikrostirnräder basiert auf einer elastischen Kunststoffschnur, deren Außendurchmesser dem Innendurchmesser der Durchgangsbohrung des Mikrostirnrades entsprechen sollte. Am Schnurende wird eine Kugel aufgeschmolzen (siehe Abbildung 4). Die Schnur fädelt man durch die Durchgangsboh- 


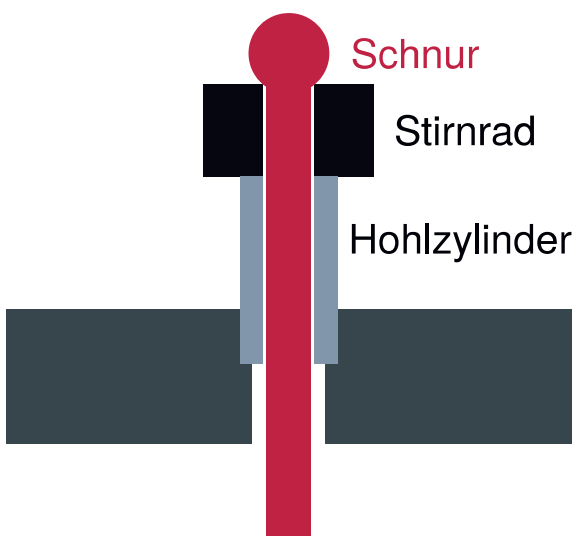

Abbildung 4: Prinzipskizze der Aufspannung für Außenverzahnungen mithilfe einer elastischen Schnur.

rung des Werkstücks und durch einen Hohlzylinder. Das Werkstück wird damit auf den Hohlzylinder zentriert (Präzision von wenigen Mikrometern) und fixiert, indem eine definierte und wiederholbare Spannkraft mithilfe von Gewichten erzeugt wird [12]. Durch die hohe Elastizität der Schnur (zum Beispiel aus Fluorcarbon) werden Spannungsspitzen, die dem Werkstück schaden können, im Normalfall vermieden (Ausnahmen können nachgiebige Werkstücke bei gleichzeitig hoher Spannkraft sein).

Die experimentell von uns erprobte Lösung für eine maschinennahe Reinigung von Werkstücken und Tastern ist das $\mathrm{CO}_{2}$-Schneestrahlen [13]. Das Reinigungsverfahren ist schnell, effektiv und maschinennah einsetzbar. Nachteile sind die tiefe Temperatur des Strahls bestehend aus festem und gasförmigen $\mathrm{CO}_{2}\left(-78^{\circ} \mathrm{C}\right.$, was zu Kondensation an Oberflächen führen kann) und der hohe Strahldruck (vergleichbar mit einer Druckluftreinigung). Eine Beschädigung von Oberflächen ist aufgrund der geringen Härte der Trockeneispartikel ausgeschlossen, weshalb dieses Reinigungsverfahren auch bei Optiken angewendet wird [14].

Mögliche Optimierungen des Reinigungsverfahrens sind gepulste Gaszufuhr, Verwendung eines Schutzgases und Reinigung mit Dry-Box. Die Eignung des Verfahrens für die Reinigung von Mikrotastern haben wir ebenfalls untersucht. Die üblichen Schwachstellen von membranbasierten Mikrotastern sind die fragilen Membranen. Hier muss verhindert werden, dass der Krafteintrag durch den Gasstrom kritisch wird. Wir konnten dies durch Anpassung der Strahlrichtung, Pulsen und Klemmung des Tasterschaftes erreichen. Einen negativen Einfluss der niedrigen Temperaturen konnten wir nicht beobachten. Je nach Materialpaarung und für spezielle Aufbauten kann dieser Aspekt allerdings kritisch sein.
Eine Schutzumhausung mit integriertem Mikroskopkamera- und Sensorsystem verbindet die Abschirmung der direkten Messumgebung mit Überwachung der Umweltparameter (Temperatur, Feuchte und Partikeldichte) und erleichtert das Einrichten der Messung.

Die Schutzumhausung besteht aus einem Aluminiumprofilrahmen, der an drei Seiten mithilfe von Acrylglas geschlossen ist (s. Abbildung 5). Die verbleibenden offenen Seiten ermöglichen den Zugang zu Drehtisch, Tastkopf und Tasterwechselbank. Aufgrund der großen Flächen und der Nähe zum Werkstück und Mikrotaster sollten die Acrylglasscheiben antistatisch beschichtet sein (ESD-Material).

Die Schutzumhausung arbeitet passiv. Aktive Filterung, Temperierung und Luftströmung haben wir zur Reduzierung von Gewicht, Kosten und Entwicklungszeit verworfen.

Die Leistungsfähigkeit der passiven Lösung wurde in Experimenten mit Partikelfallen bestätigt: Die Partikeldichte sank um $32 \%$ bei Verwendung der Schutzumhausung im Vergleich zur Standardkonfiguration der Maschine. Bei den Experimenten wurden jeweils zwei Partikelfallen aufgestellt. Eine Partikelfalle wurde maschinennah aufgestellt, um das Grundniveau der Partikelbelastung für jede Versuchsreihe zu ermitteln. Die zweite Partikelfalle platzierten wir neben dem Drehtisch der Maschine, sodass diese bei Verwendung der Schutzumhausung miteingeschlossen wird. Die Auswertung der

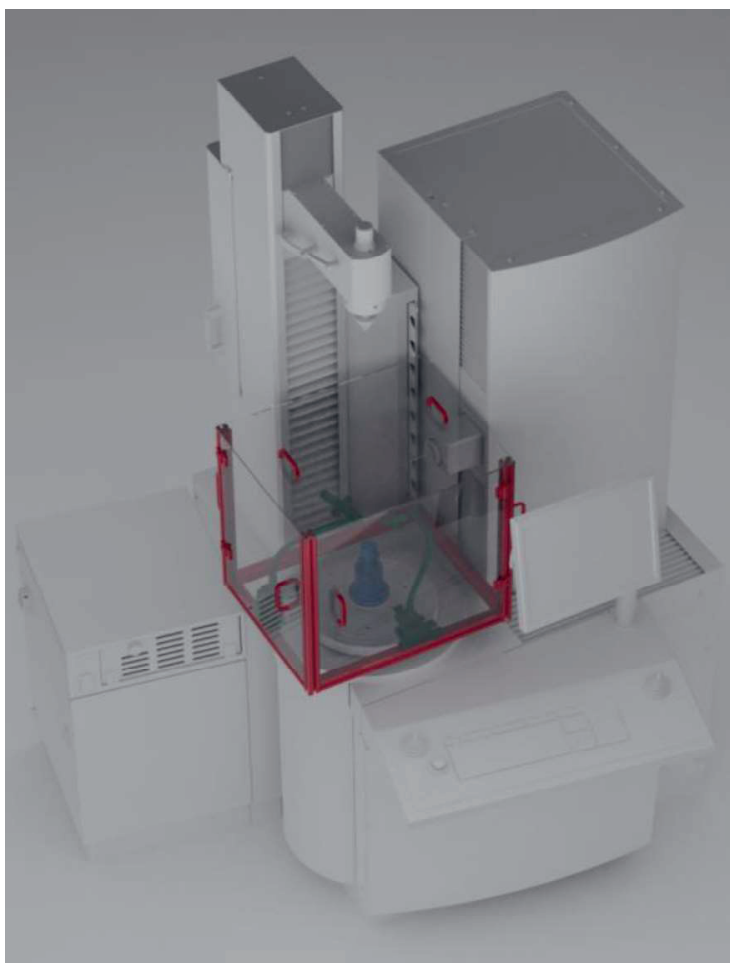

Abbildung 5: Digitales Modell des Mikroenvironments (in Farbe) integriert auf der Klingelnberg $P 40$ (in grau) 
Partikelfallen erfolgte über eine automatisierte Partikelerkennung und -analyse mithilfe eines Lichtmikroskops und dessen Software.

\section{Diskussion}

Die Messung von Mikrogeometrien ist ein wirtschaftlich wichtiger Prozess, was die Weiterentwicklung auf diesem Gebiet der Metrologie antreibt. Optische Messtechnik und dimensionelle Computertomografie haben in den letzten Jahren eine enorme Weiterentwicklung erfahren. Trotzdem ist die taktile Messtechnik oft weiterhin die beste Wahl, da sie zuverlässige, rückgeführte Resultate mit den meist geringsten Messunsicherheiten liefert. Multisensor-Koordinatenmessgeräte vereinen beispielsweise die Vorteile optischer und taktiler Technologien.

Optische Verfahren können bei Verwendung von Linien- oder Flächensensoren Vorteile bezüglich Geschwindigkeit bieten. Insbesondere die Computertomografie bietet durch die ganzheitliche Erfassung der Werkstücke (inklusive innenliegender Geometrien und Einschlüsse) großes Potenzial.

Hindernisse für die Mikromesstechnik im Allgemeinen sind die Notwendigkeit von speziellen Messverfahren und -systemen, das Fehlen von geeigneten Normalen und die hohen Anforderungen an Messunsicherheit und Zuverlässigkeit.

Neben der Weiterentwicklung von Hardware kommt der Software eine entscheidende Bedeutung zu. (Zukunfts-)Themen, die allerdings nicht nur beschränkt sind auf den Mikrobereich sind, sind Auswertealgorithmen, digitale Zwillinge, intelligente und lernende Systeme, Optimierung von Prüfplänen, Schnittstellen zum Datenaustausch, Kommunikation mit Fertigungsmaschinen oder anderer Software zur Steuerung von Unternehmensprozessen [15, 16, 17, 18, 19].

\section{Zusammenfassung}

Drei Systemkomponenten für die Mikrokoordinatenmesstechnik wurden entwickelt. Neben dem IMT-PTB-Mikrotaster haben wir ein Mikroinnenverzahnungsnormal und ein Mikroenvironment realisiert. Alle drei Komponenten zielen darauf ab, kommerzielle Koordinatenmessgeräte für die Mikromesstechnik zu erweitern. Der IMT-PTB-Mikrotaster:

- ist isotrop

- ermöglicht Auslenkungen bis zu $\pm 400 \mu \mathrm{m}$ in allen Achsen

- kann mit Tastkugeldurchmessern zwischen $50 \mu \mathrm{m}$ und $1 \mathrm{~mm}$ verwendet werden

- hat Antastkräfte von circa $30 \mathrm{mN}$ bei einer Auslenkung von $100 \mu \mathrm{m}$
Das Mikroinnenverzahnungsnormal ist ein neuartiger, rückgeführter Prüfkörper mit Messunsicherheiten im Sub-Mikrometer-Bereich. Es wurden eine Titan-Version für die Computertomografie und eine Hartmetall-Version für taktile und optische Messungen entwickelt.

Das Mikroenvironment vereint Komponenten zur Aufspannung von Mikrostirnrädern, $\mathrm{CO}_{2}-$ Schneestrahlen, Schutz vor Kontamination der direkten Messumgebung einschließlich Werkstück und Taster, Monitoring mithilfe von zwei Lichtmikroskopkameras und Erfassung von Umweltparametern (Temperatur, Feuchte, und Partikeldichte).

Die kombinierte Verwendung des Gesamtsystems soll eine hochpräzise, rückgeführte Mikroverzahnungsmessung mit kommerziellen Koordinatenmessgeräten ermöglichen.

\section{Ausblick}

Für die Zukunft planen wir die Integration des IMT-PTB-Mikrotasters in weitere Koordinatenmessgeräte wie zum Beispiel das in der PTB entwickelte Mikro-Koordinatenmessgerät [20]. Zudem streben wir eine Kommerzialisierung an. Weiterhin sind Messungen mit $50 \mu \mathrm{m}$ Tastkugeln und weitere Tasterkonfigurationen in Stern-, T- oder L-Form in Vorbereitung. Diese Tasteranordnungen ermöglichen die optimierte Messung von komplexen Mikrogeometrien.

Zur Verbesserung der Oberflächenqualität des Mikroinnenverzahnungsnormals werden Fertigungsexperimente mit Schnellarbeitsstahl (HSS) und Superfeinkorn-Hartmetall durchgeführt. Damit erhoffen wir uns eine geringere Rauheit und eine größere Oberflächenhärte, die auch höchsten Flächenpressungen aufgrund der kleinen Antastkugeln standhält [21].

\section{Danksagung}

Wir danken Michael Neugebauer, Astrid Linkogel, Fabricio Borges de Oliveira und Dieter Schulz für die Durchführung der Vergleichsmessungen und Kalibrierungen.

Wir danken der DFG für die Projektförderung (KN 1181/1-1).

Wir danken Rudolf Meeß und Christian Wolpert für die Fertigung der Prüfkörper. 


\section{Literatur}

[1] D. Metz, A. Dietzel, Mikrotaster und Verfahren zur Herstellung. Patent DE102017206145B3 https://patents.google.com/patent/DE102017206145B3/de

[2] A. A. Weckenmann, T. Estler, G. Peggs, D. McMurtry, Probing systems in dimensional metrology CIRP Annals - Manufacturing Technology 53 (2) 657-684. DOI:10.1016/S00078506(07)60034-1.

[3] D. Metz, A. Dietzel, 3-d isotropic tactile microprobe based on a silicon parallelogram kinematic: From concept to fabrication, Journal of Microelectromechanical Systems (2018) 1-14. DOI:10.1109/jmems.2018.2877926.

[4] N. Ferreira, Entwicklung, Integration und Erprobung eines piezoresistiven 3D-Mikrotasters, $\mathrm{Ph}$.D. thesis. Technische Universität Braunschweig. ISBN 978-3-8440-3546-9.

[5] D. Metz, S. Jantzen, D. Wessel, G. Mies, J. Lüdenbach, M. Stein, A. Dietzel, K. Kniel, Design of an isotropic microprobe and a microenvironment for high-precision coordinate metrology. Measurement Science and Technology, submitted (2019).

[6] D. Metz, N. Ferreira, J. Chaillot, M. Stein, K. Kniel, A. Dietzel, Integration of a piezoresistive microprobe into a commercial gear measuring instrument, Precision Engineering (2018). DOI:10.1016/j.precisioneng.2018.10.003.

[7] Klingelnberg, P 40 - Klingelnberg precision measuring center. https://www.klingelnberg.com/en/business-divisions/precision-measuring-centers/precision-measuring-centers/detail-page/product/p-40/

[8] S. Jantzen, Design of a high-precision microgear metrology system, Ph.D. thesis. Technische Universität Braunschweig. ISBN 978-395606-455-5

[9] S. Jantzen, M. Neugebauer, R. Meeß, C. Wolpert, A. Dietzel, M. Stein, K. Kniel, Novel measurement standard for internal involute microgears with modules down to $0.1 \mathrm{~mm}$. Measurement Science and Technology 29 (12) (2018) 125012. DOI:10.1088/1361-6501/aae6f4.

[10] JCGM, Evaluation of measurement data guide to the expression of uncertainty in measurement (GUM), Tech. rep., Joint Committee for Guides in Metrology (JCGM). http://www.bipm.org/en/publications/guides/gum.html

[11] S. Jantzen, M. Stein, A. Dietzel, K. Kniel, Microgear measurement standards: comparing tactile, optical and computed tomography measurements, in: AGMA's 2018 Fall Technical Meeting, 18FTM16. https://members.agma.org/ltemDetail?iProductCode=18FTM16\&Category=TECH\%20PAPER

[12] S. Jantzen, R. Meeß, M. Stein, K. Kniel, A. Dietzel, Clamping of microgears with a compliant string, in: MacroScale 2017 - Recent developments in traceable dimensional measurements, 2017. DOI:10.7795/810.20180323B

[13] S. Jantzen, T. Decarreaux, M. Stein, K. Kniel, A. Dietzel, $\mathrm{CO}_{2}$ snow cleaning of miniaturized parts, Precision Engineering 52 (2018) 122129. DOI:10.1016/j.precisioneng.2017.11.017.
[14] R. Kohli, K. L. Mittal (Eds.), Developments in Surface Contamination and Cleaning, Vol. 11, Elsevier Science, 2018.

[15] S. Boschert, R. Rosen, Digital Twin - The Simulation Aspect, Springer International Publishing, 2016, pp. 59-74. DOI:10.1007/978-3319-32156-1_5.

[16] D. Heißelmann, M. Franke, T. Kistner, C. Schwehn, K. Wendt, Determination of measurement uncertainty by simulation, in: International Conference on Advanced Mathematical and Computational Tools in Metrology and Testing (AMTCM 2017), 2017. https://arxiv.org/abs/1707.01091

[17] R. Schmitt, Das Internet of Production: Modellbasierte Qualitätssicherung in der digitalisierten Produktion, in: VDI-Berichte 2319, Messunsicherheit praxisgerecht bestimmen, 8. Fachtagung Erfurt, VDI Wissensforum GmbH, 2017.

[18] R. K. Leach, P. Bointon, X. Feng, S. Lawes, S. Piano, N. Senin, D. Sims-Waterhouse, P. Stavroulakis, R. Su, W. Syam, M. Thomas, Information-rich manufacturing metrology, in: Precision Assembly in the Digital Age, Springer International Publishing, 2018, pp. 145-157. DOI:10.1007/978-3-030-05931-6_14.

[19] D. Imkamp, R. Schmitt, J. Berthold, Blick in die Zukunft der Fertigungsmesstechnik. Technisches Messen 79 (10) 433-439. DOI:10.1524/teme.2012.0251.

[20] L. Yu, G. Molnar, S. Bütefisch, C. Werner, R. Meeß, H.-U. Danzebrink, J. Flügge, Micro coordinate measurement machine (uCMM) using voice coil actuator with interferometric position feedback, in: J. Tan, J. Lin (Eds.), Tenth International Symposium on Precision Engineering Measurements and Instrumentation, SPIE. DOI:10.1117/12.2511527.

[21] E. J. C. Bos, Aspects of tactile probing on the micro scale. Precision Engineering 35 (2) 228240. DOI:10.1016/j.precisioneng.2010.09.010. 